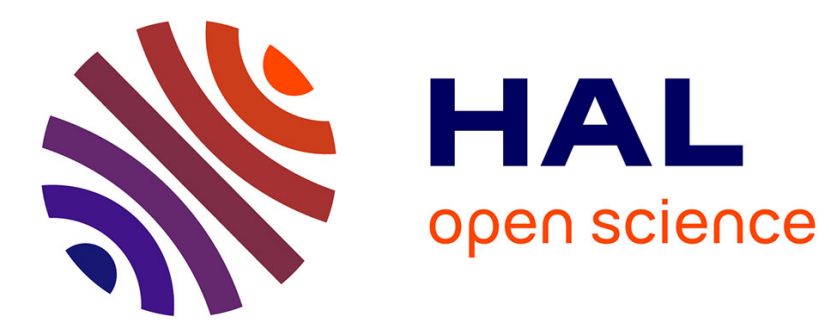

\title{
Passivation par l'hydrogène de défauts recombinants dans les photopiles réalisées sur rubans de silicium polycristallin RAD
}

\author{
M. Mautref, C. Lacroix, C. Belouet, C. Fages, B. Biotteau, F. Amoult
}

\section{To cite this version:}

M. Mautref, C. Lacroix, C. Belouet, C. Fages, B. Biotteau, et al.. Passivation par l'hydrogène de défauts recombinants dans les photopiles réalisées sur rubans de silicium polycristallin RAD. Revue de Physique Appliquée, 1984, 19 (4), pp.333-342. 10.1051/rphysap:01984001904033300 jpa-00245201

\author{
HAL Id: jpa-00245201 \\ https://hal.science/jpa-00245201
}

Submitted on 1 Jan 1984

HAL is a multi-disciplinary open access archive for the deposit and dissemination of scientific research documents, whether they are published or not. The documents may come from teaching and research institutions in France or abroad, or from public or private research centers.
L'archive ouverte pluridisciplinaire HAL, est destinée au dépôt et à la diffusion de documents scientifiques de niveau recherche, publiés ou non, émanant des établissements d'enseignement et de recherche français ou étrangers, des laboratoires publics ou privés. 


\title{
Passivation par l'hydrogène de défauts recombinants dans les photopiles réalisées sur rubans de silicium polycristallin RAD
}

\author{
M. Mautref, C. Lacroix (*), C. Belouet, C. Fages, B. Biotteau et F. Arnoult \\ Laboratoires de Marcoussis, Centre de Recherches de la C.G.E., Route de Nozay, 91460 Marcoussis, France \\ ${ }^{*}$ ) Centre Universitaire de Clermont-Ferrand, 63170 Aubière, France
}

(R̀eçu le 2 novembre 1983, révisé le 12 janvier 1984, accepté le 17 janvier 1984)

\begin{abstract}
Résumé. - Cet article présente les résultats d'une étude exploratoire de la passivation par l'hydrogène des défauts recombinants dans les rubans de silicium polycristallin élaborés par la méthode RAD. L'incorporation d'hydrogène a été effectuée sur des photopiles nues au moyen d'une source d'ions du type Kaufman. On montre que les caractéristiques globales des photopiles - photocourant, tension de circuit ouvert et facteur de forme - sont considérablement améliorées tandis que leurs distributions sont resserrées. Ces améliorations sont reliées à la réduction de la vitesse de recombinaison des porteurs minoritaires sur les interfaces du type joints de grains ou de mâcles et surtout, à l'augmentation de la longueur de diffusion dans les parties homogènes; ce dernier effet est associé à une réduction importante de l'activité des centres pièges. Ainsi, le rendement de conversion, sous éclairement $A M 1$, des photopiles RAD « passivées » à l'hydrogène peut être relativement élevé : la meilleure photopile ainsi traitée présente un rendement de $15,5 \%$ (surface active, $3 \mathrm{~cm}^{2}$ ).
\end{abstract}

\begin{abstract}
Results of early hydrogen passivation studies of defects in polycrystalline silicon ribbons grown by the RAD process are presented. Hydrogen was incorporated in uncoated solar cells by means of a Kaufman ion source. It is shown that the solar cell characteristics, namely : the photocurrent density, the open-circuit voltage and the fill factor - are considerably improved whereas their distributions are narrowed. These improvements are ascribed to an increase of the minority-carrier diffusion length in the bulk rather than to a reduction of the recombination velocity of minority-carriers at interfaces - grain or twin boundaries - ; the bulk passivation goes along with an important reduction of the minority-carrier trap activity. Thus, the AM1 conversion efficiency of hydrogenpassivated RAD solar cells may be relatively high. The best cell thus treated had a conversion efficiency of $15.5 \%$ (active area, $3 \mathrm{~cm}^{2}$ in size).
\end{abstract}

\section{Introduction.}

L'élaboration de silicium sous forme de ruban est une approche prometteuse en vue d'abaisser le coût des photopiles pour applications terrestres [1]. Dans ce contexte, de nombreuses méthodes de croissance de rubans ont été développées [2]. Parmi celles-ci, le procédé RAD dont le principe est rappelé dans la figure 1 consiste à cristalliser un film de silicium sur chaque face d'un ruban de carbone souple [3]; les couches de silicium opposées sont ensuite séparées du support en carbone par brûlage de celui-ci à haute température. Elles sont constituées de grains de grandes dimensions très allongés dans le sens du tirage. Ces grains, dont la largeur caractéristique est de plusieurs millimètres, sont généralement en relations de mâcles, les joints de grains étant perpendiculaires à la surface de la couche.
Les caractéristiques des photopiles préparées sur ce matériau sont affectées aussi bien par les défauts intragranulaires étendus - joints de mâcles $\Sigma 3$ imparfaits, sous-joints et lignes de dislocations -, que par les effets de pièges dans les parties homogènes [3-5].

De nombreux travaux ont montré que la passivation chimique par fixation d'hydrogène permettait de réduire, voire supprimer, l'activité électrique des interfaces recombinantes [6-8]. Cet effet de passivation a aussi été mis en évidence dans les parties homogènes, exemptes de défauts étendus [9]. Enfin, des améliorations importantes des performances des photopiles ont été observées après incorporation d'hydrogène au moyen d'un plasma $\mathrm{RF}$ ou d'une source d'ions $\mathrm{H}^{+}$ du type Kaufman [8-10].

Cet article présente les résultats d'une étude exploratoire de la passivation par l'hydrogène de défauts 
recombinants dans les photopiles RAD au moyen d'une source Kaufman.

\section{Méthodes expérimentales.}

Les études d'hydrogénation ont été effectuées sur des photopiles du type $\mathrm{n}^{+} / \mathrm{p}$ obtenues par diffusion au phosphore à $850^{\circ} \mathrm{C}$ et dont la résistivité de la base était de 1 à $2 \Omega . \mathrm{cm}$; la profondeur de jonction était de l'ordre de $0,5 \mu \mathrm{m}$. Le contact $\mathrm{p}^{+}$sur la face arrière était obtenu par évaporation d'aluminium suivie d'un recuit rapide à $660^{\circ} \mathrm{C}$ sous atmosphère neutre tandis que le contact face avant était constitué d'une grille réalisée par évaporations successives à $150^{\circ} \mathrm{C}$ de titane, palladium et argent.

Les implantations d'hydrogène (IMPL-H) ont été effectuées au moyen d'une source d'ions $\mathrm{H}^{+}$du type Kaufman (micro-etch 3" de VEECO), sur photopiles nues (sans antireflet). Dans ces essais, la pression du plasma hydrogène était de l'ordre de $2 \times 10^{-4}$ torr et la température des échantillons était de l'ordre de $170{ }^{\circ} \mathrm{C}$; la tension d'accélération et la densité de courant faisceau étaient de $1,4 \mathrm{kV}$ et $1,4 \mathrm{~mA} / \mathrm{cm}^{2}$ respectivement tandis que la durée d'implantation était de $2 \mathrm{~min}$. Certaines photopiles avaient au préalable subi un recuit à basse température $\left(430^{\circ} \mathrm{C}\right)$, noté RBT-430, d'une durée de $30 \mathrm{~min}$.

Les caractéristiques $I-V$ sous éclairement AM1 (ou fraction de AM1) et à l'obscurité ont été systématiquement mesurées sur les photopiles brutes, après RBT-430 et après implantation hydrogène (IMPL-H). Les tracés $I-V$ à l'obscurité ont été realisés dans la gamme -196 à $+100{ }^{\circ} \mathrm{C}$ au moyen d'une chaîne de mesure pilotée par un micro-ordinateur. Enfin les études au microscope à balayage (MEB) en mode EBIC ont permis de suivre l'évolution des propriétés recombinantes des interfaces alors que les balayages des échantillons en mode LBIC (source laser YAG : Nd à $1,06 \mu \mathrm{m}$ ) ont permis de suivre les variations de la longueur de diffusion, $L n$, des porteurs minoritaires dans les parties homogènes de la base.

\section{Résultats.}

3.1 AMÉLIORATION DES CARACTÉRISTIQUES DES PHOTOPILES. - D'une manière générale, le traitement IMPLH effectué sur les photopiles nues améliore leurs caractéristiques : tension de circuit ouvert $V_{\text {co }}$, densité de photocourant $J_{\mathrm{ph}}\left({ }^{1}\right)$, facteur de forme FF et rendement de conversion $\eta$. L'étude des distributions de ces caractéristiques avant et après IMPL-H met en évidence une augmentation de leurs valeurs moyennes et une réduction de la largeur de leurs distributions. Ceci apparaît dans le tableau I qui présente les caractéristiques de photopiles RAD de dimension $1 \times 2 \mathrm{~cm}$ réalisées à partir d'un ruban obtenu à une vitesse de tirage, $u$, de $10 \mathrm{~cm} / \mathrm{min}$. On note des augmentations

( ${ }^{1}$ Les densités de photocourant sont données pour la surface active des photopiles.
Tableau I. - Caractéristiques des photopiles - sans antireflet $-d u$ ruban $\mathrm{F}$.

[Characteristics of solar cells - not A. R. coated of ribbon $F$.]

\begin{tabular}{|c|c|c|c|c|c|c|c|c|c|c|c|c|}
\hline \multirow{2}{*}{$\begin{array}{l}\text { RUBAN F } \\
N^{*} \\
\end{array}$} & \multicolumn{4}{|c|}{ BRUT } & \multicolumn{4}{|c|}{ RBT 430} & \multicolumn{4}{|c|}{ IMPL-H } \\
\hline & $\overline{j p h}$ & $v_{\infty}$ & FF & $\eta$ & Jph & Voo & $\mathbf{F F}$ & $\bar{\eta}$ & $\overline{\mathrm{jph}}$ & Voo & $\mathbf{F F}$ & $\eta \mathrm{s}$ \\
\hline $\begin{array}{l}6 \\
7 \\
8 \\
9 \\
10 \\
11 \\
11 \\
13 \\
13 \\
14\end{array}$ & $\begin{array}{l}18,2 \\
18,6 \\
18,1 \\
11,6 \\
17,2 \\
18,5 \\
17,3 \\
17,3 \\
18,7 \\
16,1 \\
17,9 \\
17,9 \\
17,5 \\
17,9 \\
19,2\end{array}$ & 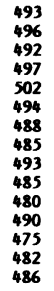 & 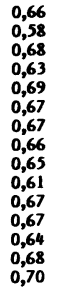 & $\begin{array}{l}5,9 \\
5,3 \\
6,1 \\
5,8 \\
5,9 \\
6,91 \\
5,7 \\
5,5 \\
6,0 \\
4,7 \\
518 \\
5,9 \\
5,3 \\
,, 8 \\
6,5\end{array}$ & $\begin{array}{l}18,2 \\
20,3 \\
19,7 \\
19,7 \\
18,3 \\
19,4 \\
18,9 \\
19,6 \\
20,6 \\
18,0 \\
19,6 \\
20,5 \\
20,0 \\
21,3 \\
19,6\end{array}$ & $\begin{array}{l}502 \\
505 \\
506 \\
510 \\
503 \\
505 \\
502 \\
506 \\
507 \\
500 \\
500 \\
506 \\
498 \\
504 \\
500\end{array}$ & $\begin{array}{l}0,66 \\
0,068 \\
0,066 \\
0,66 \\
0,066 \\
0,067 \\
0,69 \\
0,71 \\
0,066 \\
0,068 \\
0,68 \\
0,067 \\
0,68 \\
0,72\end{array}$ & $\begin{array}{l}6,0 \\
6,9 \\
6,7 \\
6,6 \\
6,1 \\
6,6 \\
6,5 \\
7,0 \\
6,9 \\
6,0 \\
6,7 \\
7,1 \\
6,6, \\
7,3 \\
7,0\end{array}$ & $\begin{array}{l}19,6 \\
21,7 \\
21,7 \\
21,5 \\
11,0 \\
20,1 \\
20,8 \\
21,8 \\
22,4 \\
19,6 \\
21,9 \\
22,9 \\
22,2 \\
22,2 \\
22,0 \\
22,4\end{array}$ & $\begin{array}{l}5225 \\
527 \\
530 \\
531 \\
526 \\
527 \\
530 \\
530 \\
530 \\
528 \\
533 \\
531 \\
527 \\
529 \\
528\end{array}$ & $\begin{array}{l}0,67 \\
0,72 \\
0,71 \\
0,70 \\
0,767 \\
0,70 \\
0,73 \\
0,72 \\
0,768 \\
0,717 \\
0,72 \\
0,72 \\
0,769 \\
0,72 \\
0,73\end{array}$ & 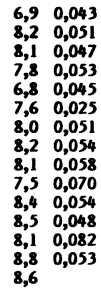 \\
\hline $\begin{array}{l}16 \\
17 \\
18 \\
19 \\
20 \\
21 \\
22 \\
23 \\
24\end{array}$ & $\begin{array}{l}19,7 \\
17,8 \\
19,8 \\
18,2 \\
18,9 \\
18,3 \\
19,0 \\
18,5 \\
20,4 \\
18,2 \\
18,4 \\
18,7 \\
17,2\end{array}$ & $\begin{array}{l}498 \\
486 \\
484 \\
471 \\
493 \\
493 \\
495 \\
483 \\
4493 \\
4992 \\
488 \\
488 \\
476\end{array}$ & $\begin{array}{l}0,69 \\
0,65 \\
0,67 \\
0,63 \\
0,65 \\
0,69 \\
0,65 \\
0,67 \\
0,66 \\
0,70 \\
0,70 \\
0,69 \\
0,66\end{array}$ & $\begin{array}{l}6,8 \\
5,6 \\
6,4 \\
5,4 \\
6,1 \\
6,2 \\
6,1 \\
6,0 \\
6,6 \\
6,6 \\
6,3 \\
6,3 \\
5,4\end{array}$ & & & & & $\begin{array}{l}20,9 \\
21,5 \\
23,0 \\
21,4 \\
21,7 \\
21,7 \\
21,2 \\
21,2 \\
21,8 \\
19,5 \\
20,3 \\
21,8 \\
20,0\end{array}$ & $\begin{array}{l}528 \\
528 \\
5224 \\
527 \\
530 \\
533 \\
533 \\
530 \\
554 \\
532 \\
530 \\
527\end{array}$ & $\begin{array}{l}0,72 \\
0,72 \\
0,70 \\
0,69 \\
0,67 \\
0,72 \\
0,69 \\
0,71 \\
0,69 \\
0,72 \\
0,73 \\
0,73 \\
0,72\end{array}$ & $\begin{array}{lll}7,9 & 0,038 \\
7,8 & 0,044 \\
8,5 & 0,064 \\
7,8 & 0,037 \\
7,6 & 0,045 \\
8,0 & 0,060 \\
7,8 & 0,045 \\
7,90,043 & 0,043 \\
7,9 & 0,043 \\
7,3 & 8,1 \\
8,1 & \\
8,1 & \\
7,5 & \end{array}$ \\
\hline
\end{tabular}

\begin{tabular}{lllllllllllll}
\hline$\overline{\mathbf{x}}$ & 18,2 & 488 & 0,663 & 5,9 & 19,6 & 504 & 0,673 & 6,64 & 21,2 & $528,60,707$ & 7,9 & 0,050 \\
\hline$\sigma$ & 0,9 & 7,3 & 0,028 & 0,45 & 1,0 & 3,2 & 0,014 & 0,40 & 1,0 & 2,3 & 0,019 & $0,450,01$ \\
\hline
\end{tabular}

substantielles des valeurs moyennes de $\bar{V}_{c 0}\left(\Delta \bar{V}_{\mathrm{co}}=\right.$ $40 \mathrm{mV}, \bar{J} \quad\left(\Delta \bar{J}=3 \mathrm{~mA} \mathrm{~cm}{ }^{2}\right.$ et $\left.\overline{F F} \Delta \overline{F F}=0,044\right)$ qui se traduisent par un gain de $33,8 \%$ sur le rendement. de conversion AM1 moyen des photopiles brutes d'élaboration.

Les écarts types, $\sigma$, sur ces caractéristiques font apparaître un resserrement des distributions après IMPL-H particulièrement significatif pour $V_{\text {co }}$ et $F F$; par contre la largeur de la distribution de $J_{\mathrm{ph}}$, bien que mieux centrée, reste inchangée (Figs. 2 à 5 ).

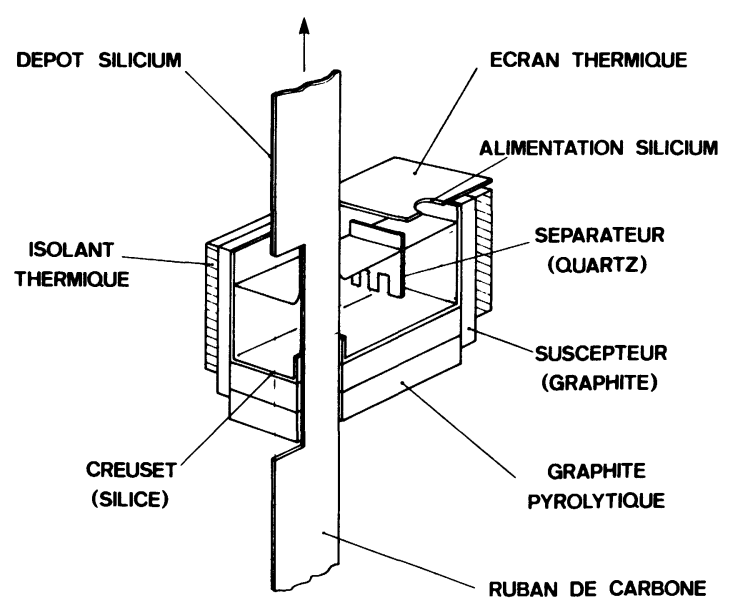

Fig. 1. - Schéma de principe du procédé RAD.

[Schematic of the RAD growth process.] 


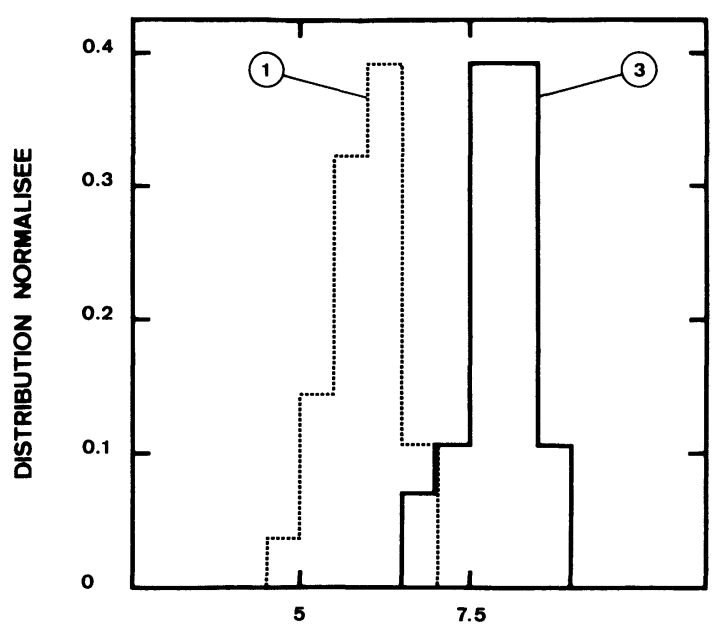

RENDEMENT DE CONVERSION $\eta(\%)$

Fig. 2. - Distribution normalisée du rendement de conversion, $\eta$, sous éclairement AM1 des photopiles nues du tableau I, brutes de préparation (1) et après IMPL-H (3).

[Normal distribution of conversion efficiency, $\eta$, under AM1 illumination of uncoated cells of table I, virgin (1), and after IMPL-H (3).]

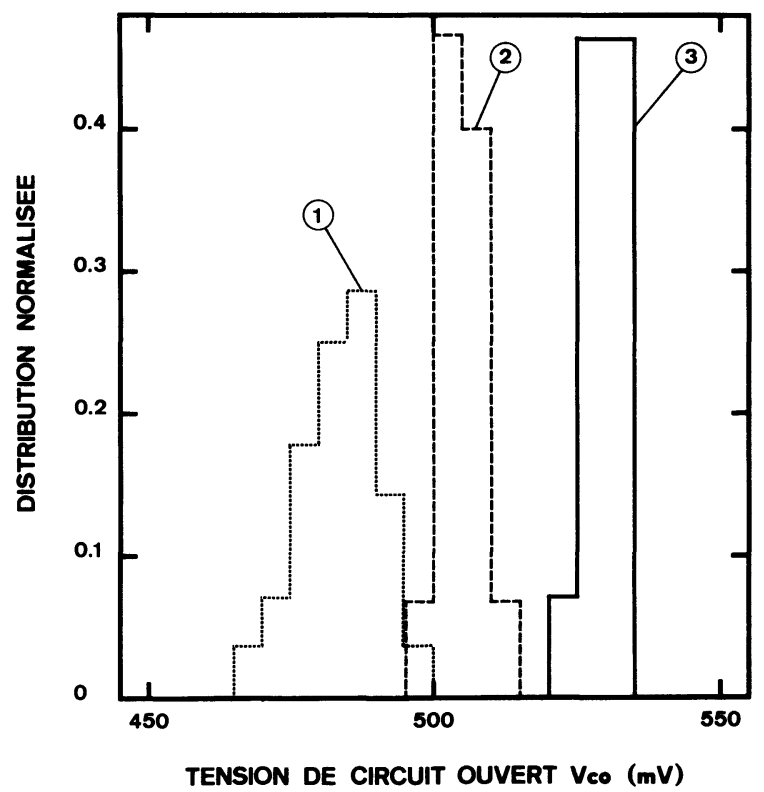

Fig. 3. - Distribution normalisée de la tension de circuit ouvert $V_{\text {cos }}$, sous éclairement AM1 des photopiles nues du tableau I, brutes de préparation (1) après RBT-430 (2) et après IMPL-H (3).

[Normal distribution of open-circuit voltage, $V_{\text {oc }}$, under AM1 illumination of uncoated cells of table I, virgin (1), after RBT-430 (2) and IMPL-H (3).]

Ainsi, d'un point de vue phénoménologique, l'effet du traitement IMPL-H est analogue à celui des recuits à basse température $\left(430^{\circ} \mathrm{C}, \mathrm{RBT}-430\right)$ effectués sur photopiles nues [11]. Cependant, la comparaison des

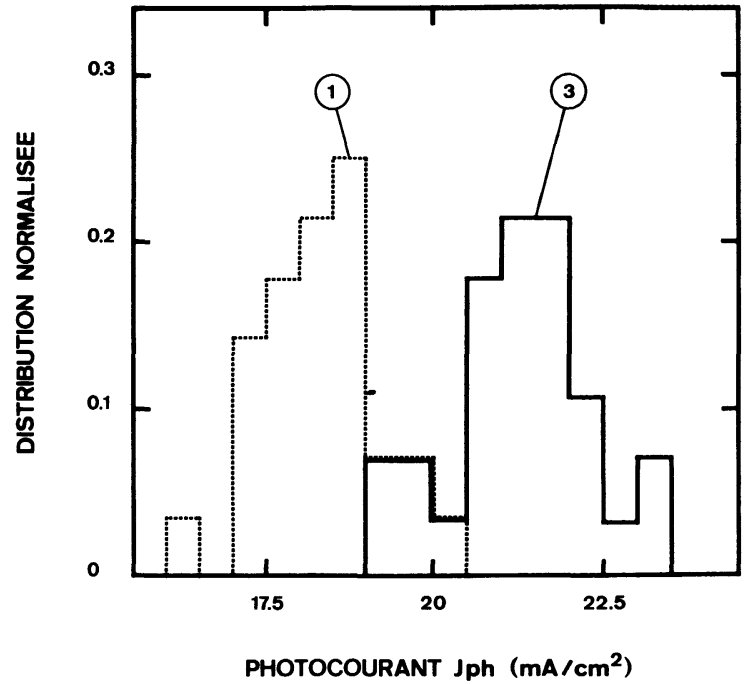

Fig. 4. - Distribution normalisée de la densité de photocourant, $J_{\text {ph }}$, sous éclairement AM1 des photopiles nues du tableau I, brutes de préparation (1) et après IMPL-H (3).

[Normal distribution of photocurrent density, $J_{\mathrm{ph}}$, under AM1 illumination of uncoated cells of table I, virgin (1) and after IMPL-H (3).]

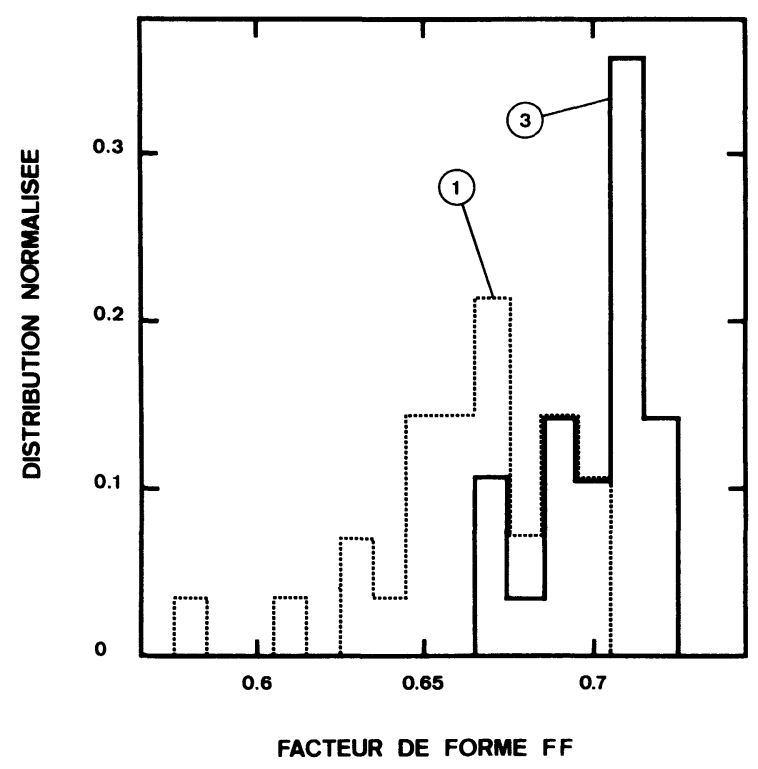

Fig. 5. - Distribution normalisée du facteur de forme, $F F$, sous éclairement AM1 des photopiles nues du tableau I, brutes de préparation (1) et après IMPL-H (3).

[Normal distribution of fill factor, $F F$, under AM1 illumination of uncoated cells of table I, virgin (1) and after IMPLH (3).]

propriétés des photopiles 1 à 15 du tableau I, après RBT-430 et IMPL-H appliqués dans cet ordre, met en évidence la plus grande efficacité du traitement hydrogène. 
Il apparaît aussi dans ce tableau que les performances des photopiles après IMPL-H sont indépendantes du traitement RBT-430 intermédiaire, qui pourrait donc être supprimé, les caractéristiques des photopiles des séries 1 à 15 et 16 à 28 (non soumises à RBT-430) n'étant pas significativement différentes (Tableau II).

Ces résultats, comparables à ceux observés dans le cas de photopiles préparées sur ruban auto-supporté EFG [12], sont transposables à l'ensemble des photopiles réalisées sur ruban RAD. Cependant, les gains sur les caractéristiques sont très variables d'un ruban à l'autre et, comme dans l'exemple ci-dessus, les gains de densité de photocourant sont toujours très dispersés. Le tableau III présente les caractéristiques typiques des photopiles préparées sur trois rubans notés $\mathrm{C}, \mathrm{E}$ et $\mathrm{F}$ élaborés à des vitesses de tirage, $u$, de 5,7 et $10 \mathrm{~cm} / \mathrm{min}$ respectivement.

Tableau II. - Valeur moyenne et écart type des caractéristiques des photopiles après JMPL-H soumises ( 1 à 15) et non soumises (16 à 28) à un traitement RBT-430 intermédiaire. Les échantillons 1 à 28 sont ceux du tableau I.

[Mean values and standard deviations of solar cells characteristics afterJMPL-H, submitted (1 to 15) and not submitted (16 to 28$)$ to an intermediate RBT-430 treatment. Samples 1 to 28 refer to table I.]

\begin{tabular}{|c|c|c|c|c|c|c|}
\hline \multirow{2}{*}{$\frac{\text { RUBAN } F}{\text { ECH. }}$} & \multicolumn{6}{|c|}{ CARACIERISTIQUES } \\
\hline & $\operatorname{sph}\left(\mathrm{man} / \mathrm{cm}^{2}\right)$ & $x \in O(m V)$ & $\mathrm{FF}$ & & $\eta x$ & s \\
\hline $1-15$ & $21,34(1,14)$ & $528,7(1,95)$ & 0.706 & $(0,020)$ & $7,97(0,57)$ & $0,052(0,013)$ \\
\hline $16-28$ & $21.1 \quad(0,87)$ & $528,6(2,7)$ & 0.708 & $(0,019)$ & $7,86(0,30)$ & $0,047(0,010)$ \\
\hline
\end{tabular}

Tableau III. - Caractéristiques . des photopiles $\left(2\right.$ ou $\left.4 \mathrm{~cm}^{2}\right)$ de trois rubans $\mathrm{C}, \mathrm{E}$ et $\mathrm{F}$. Les symboles $\bar{x}$ et $\sigma$ représentent la valeur moyenne et l'écart type de chaque caractéristique; la colonne A. $\mathrm{R}$. se rapporte aux photopiles avec antireflet.

[Solar cell $\left(2\right.$ or $\left.4 \mathrm{~cm}^{2}\right)$ characteristics of three ribbons noted C, E and F. Symbols $\bar{x}$ and $\sigma$ denote the mean value and the standard deviation of each characteristic respectively. Column A. R. refers to A. R. coated cells.]

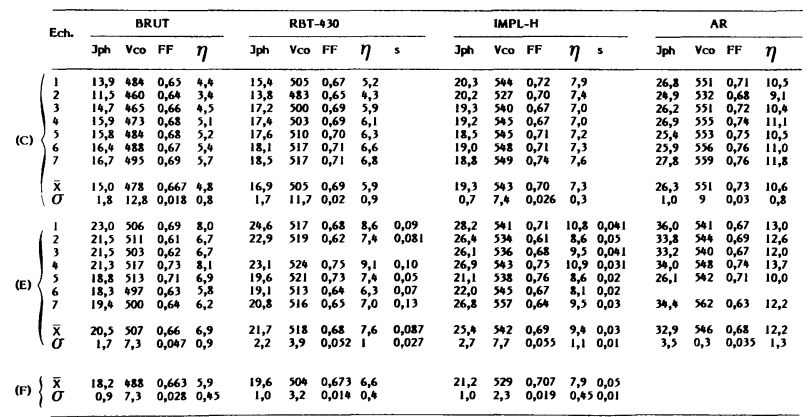

Le grande disparité des gains sur $J_{\mathrm{ph}}$ apparaît clairement dans ce tableau, le gain moyen le plus fort étant obtenu avec le ruban $\mathrm{E}$ qui présente pourtant la valeur moyenne de photocourant initial, $\bar{J}_{\mathrm{ph}}(\mathrm{i})$, la plus élevée. Les incréments $\Delta V_{\mathrm{co}}$ sont dans tous les cas une fonction décroissante et quasi-linéaire de la tension de circuit ouvert initiale $V_{\text {co }}(i)$. Les valeurs de la tension de circuit ouvert initiale extrapolées à $\Delta V_{\text {co }}=0,\left(V_{\text {co }}(\mathrm{ext})\right)$ par la méthode des moindres carrés, sont plus grandes que leurs équivalentes après RBT-430 (Figs. 6 à 8). $V_{\text {co }}$ (ext) varie significativement d'un ruban à l'autre et atteint pour certains rubans des valeurs proches de celles obtenues sur monocristal $\mathrm{Cz}$ diffusé dans les mêmes conditions $\left(V_{\text {co }}(\right.$ ext $) \sim 577 \mathrm{mV}$ sans anti-reflet pour le ruban $\mathrm{D})$.

Les variations de $J_{\text {ph }}$ et $V_{\text {co }}$ après IMPL-H ont pu être reliées directement à la réduction de l'activité de centres pièges non caractérisés jusqu'ici. La sensibilité spectrale $S_{(\lambda)}$ des photopiles RAD est en général une fonction supralinéaire du flux incident, $\phi$. Comme dans le cas des matériaux $\mathrm{Cz}$ [13], elle est approximable pour chaque longueur d'onde par la relation (1) :

$$
S_{(\lambda)}=K_{(\lambda)} \times \phi_{(\lambda)}^{(1+s(\lambda))}
$$

où $K_{(\lambda)}$ est un facteur constant et $s_{(\lambda)}$ un exposant qui traduit la non-linéarité. Les études de cet effet de saturation des pièges décrites ci-dessous ont été conduites au moyen de la mesure de la densité de photocourant sous éclairement polychromatique (éelairement AM1, délivré par un simulateur solaire, atténué dans un rapport de 1 à 10). Pour les mêmes raisons que ci-dessus, $J_{\text {ph }}$ et $\phi$ sont reliés par une

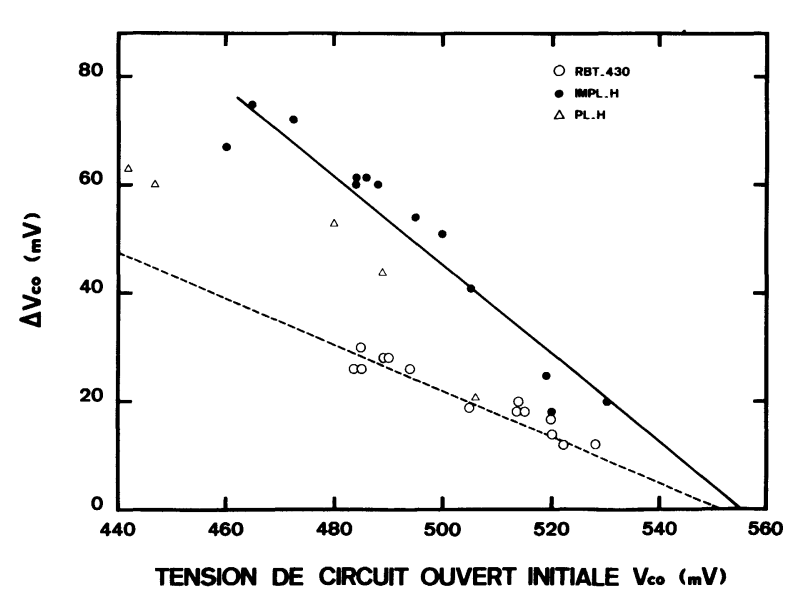

Fig. 6. - Incrément de tension de circuit ouvert $\Delta V_{\text {co }}$ en fonction de la tension initiale $V_{\text {co, }}$ après RBT-430 (O) et après IMPL-H $(\Theta)$; les triangles $(\triangle)$ se rapportent aux hydrogénations sous plasma $\mathrm{RF}$ à $430^{\circ} \mathrm{C}$ (PLH-430). Ces résultats se rapportent au ruban $\mathrm{C}$.

[Open-circuit voltage increase, $\Delta V_{o c}$, against the initial open-circuit voltage, $V_{\text {oc }}$, following an RBT-430 (open circles) and an IMPL-H (full circles); open triangles refer to hydrogenation experiments conducted with an RF plasma at $430^{\circ} \mathrm{C}$ (PLH 430). Results refer to ribbon C.] 


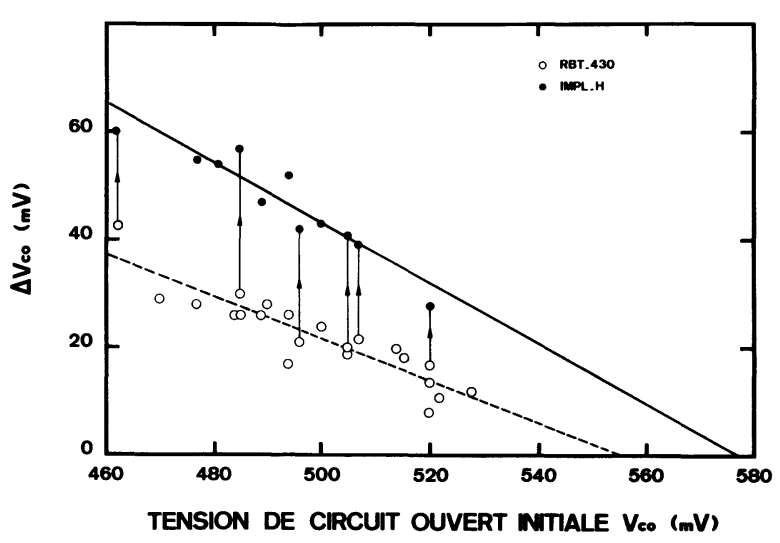

Fig. 7. - Incrément de tension de circuit ouvert en fonction de la tension initiale $V_{\text {co }}$ après RBT-430(O) et après IMPL-H (๑); les flèches indiquent les incréments obtenus après traitement IMPL-H de photopiles préalablement traitées RBT-430. Ces résultats se rapportent au ruban D.

[Open-circuit voltage increase, $\Delta V_{\text {oc }}$, against the initial open-circuit voltage, $V_{\text {oc }}$, following an RBT-430 (open circles) and IMPL-H (full circles); arrows show $V_{\text {oc }}$ increases obtained after an IMPL-H treatment of cells already RBT430 treated. Results refer to ribbon D.]

relation supralinéaire :

$$
J_{\mathrm{ph}}=K \times \phi^{(1+s)} .
$$

La comparaison des valeurs de $V_{\text {co }}, J_{\text {ph }}$ et $s$ avant et après IMPL-H montre que les incréments $\Delta V_{\text {co }}$ et $\Delta J_{\mathrm{ph}}$ sont individuellement corrélés à une réduction de $s$, c'est-à-dire, de l'activité des pièges à porteurs minoritaires [13]. Un comportement analogue, mais d'amplitude moindre, avait déjà été rapporté suite aux traitements RBT-430 et PLH-430 ( ${ }^{2}$ [ [11]. Les réductions de $s$ consécutives au traitement IMPL-H varient selon les rubans, les valeurs de $s$ les plus faibles conduisant statistiquement aux valeurs de $V_{\text {co }}$ et $J_{\mathrm{ph}}$ les plus élevées. Dans le cas du seul ruban $\mathrm{C}$ du tableau III, les valeurs de $V_{\text {co }}$ (ext) (et de $J_{\text {ph }}$ (ext) défini comme $V_{\text {co }}\left(\right.$ ext) en dépit des fortes dispersions de $J_{\text {ph }}$ ) ont été trouvées identiques pour les traitements IMPL-H et RBT-430; dans ce cas particulier, elles correspondaient à des valeurs de $s$ voisines de zéro. Par contre, des écarts importants sont observés pour la plupart des rubans.

Les études de caractéristiques $I-V$ à l'obscurité et de réponses spectrales $S(\lambda)$ des photopiles avant et après IMPL-H indiquent que les incréments $\Delta V$ co sont dus à une réduction du courant de diffusion, $I_{02}$, et à l'augmentation de la résistance parallèle, $\boldsymbol{R}_{\mathrm{p}}$, de l'homojonction; une réduction importante du courant de recombinaison $I_{01}$ a aussi été observée (Fig. 9). Cependant, les variations de $I_{01}$ proprement dit sont souvent masquées par une contribution importante de la périphérie de la jonction au courant $I$ total, comme le montre l'exemple de la figure 10 .

$\left({ }^{2}\right)$ Passivation hydrogène sous plasma R.F. à $430^{\circ} \mathrm{C}$.

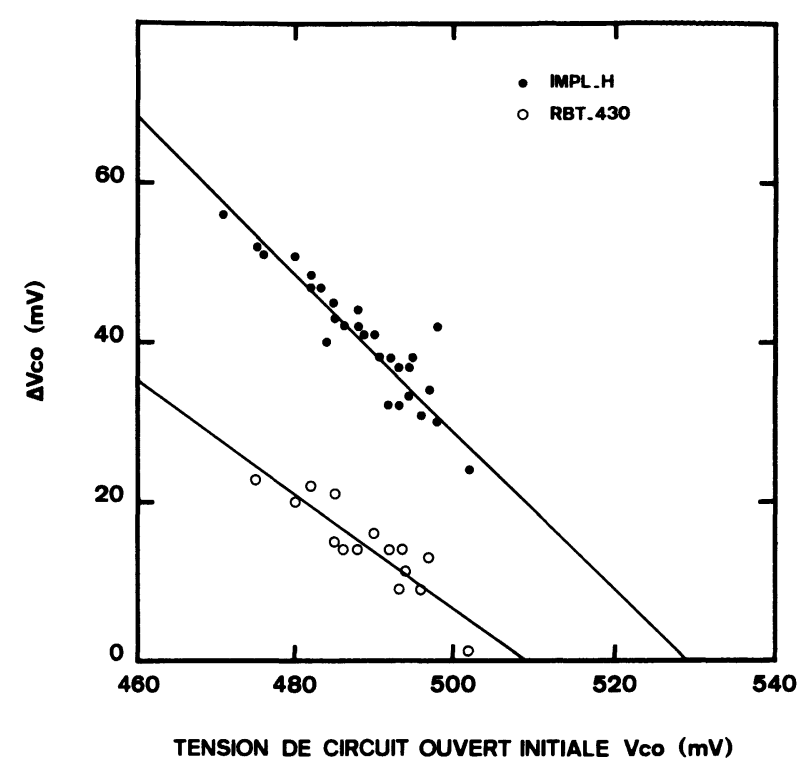

Fig. 8. - Incrément de tension de circuit ouvert en fonction de la tension initiale $V_{\text {co, }}$, après RBT- $430(0)$ et après IMPL-H (๑). Ces résultats se rapportent au ruban $\mathrm{F}$.

[Open-circuit voltage increase, $\Delta V_{\text {oc }}$, against the initial open-circuit voltage, $V_{\text {oc }}$, following an RBT-430 (open circles) and an IMPL-H (full circles). Results refer to ribbon F.]

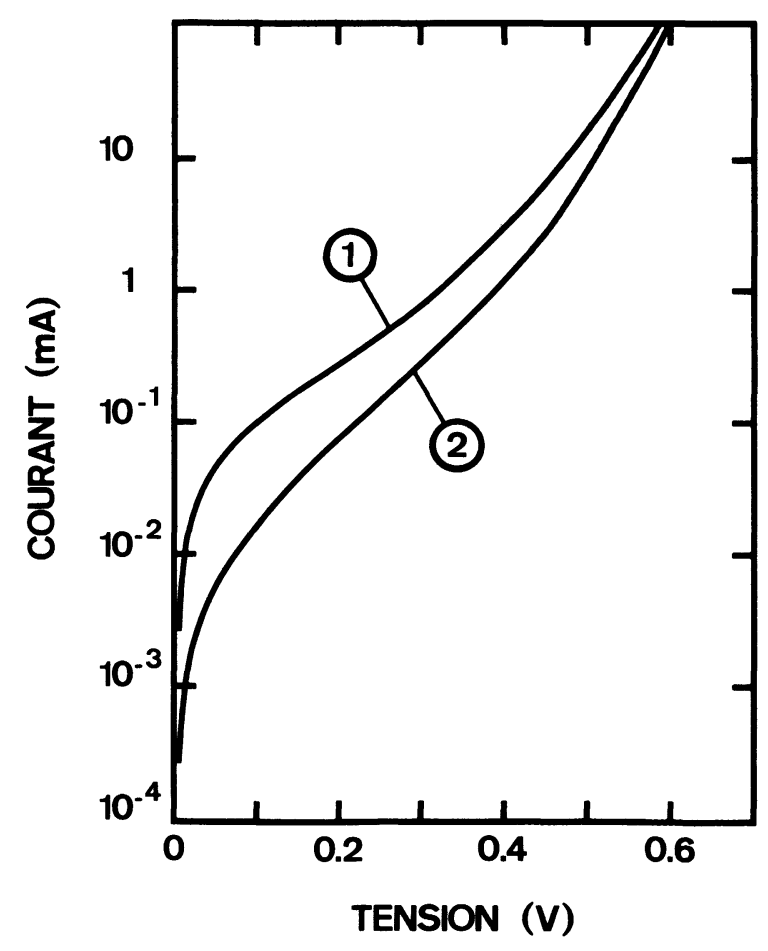

Fig. 9. - Courant d'obscurité (direct) en fonction de la tension en échelle semi-logarithmique - photopile d'origine (1) et après IMPL-H (2).

[Dark, forward $I-V$ curves on a semi-logarithmic plot (1) : virgin cell, (2) : IMPL-H treated cell.] 


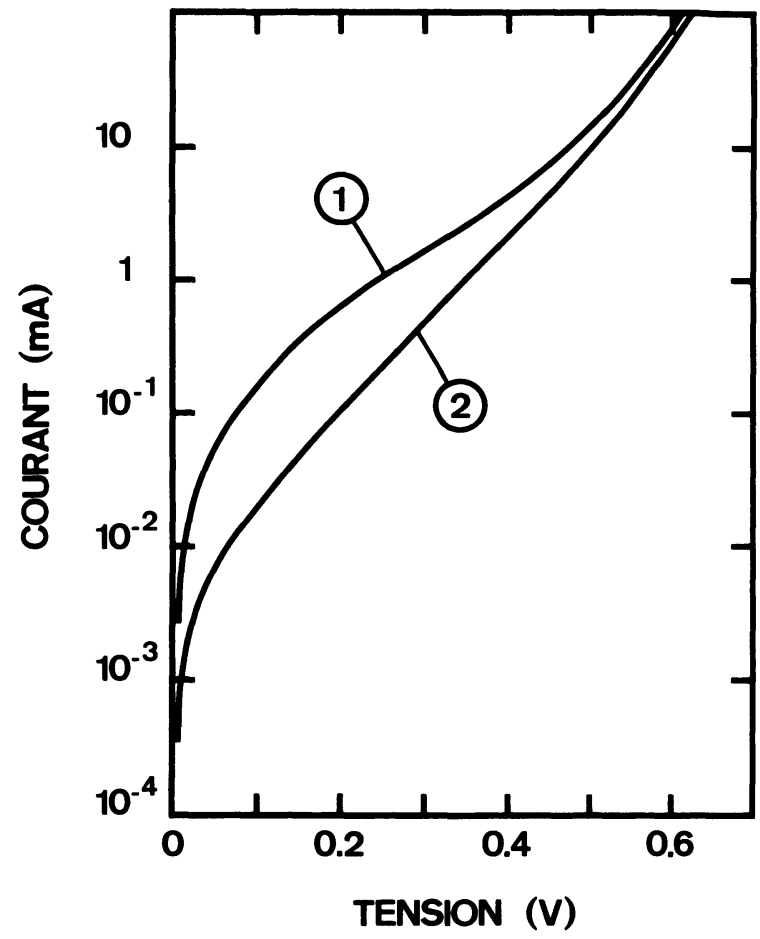

Fig. 10. - Courant d'obscurité (direct) en fonction de la tension en échelle semi-logarithmique - photopile d'origine (1) et après décapage chimique de la périphérie (2).

[Dark, forward $I-V$ curves on a semi-logarithmic plot (1) : virgin cell, (2) : same cell after edge etch.]

(Cet effet est attribué au mode de découpe par laser YAG : Nd, non optimisé pour le traitement d'échantillons minces et de planéité insuffisante.) La réduction de $I_{02}$ et l'augmentation de $J_{\mathrm{ph}}$ traduisent surtout l'augmentation de la longueur de diffusion des porteurs minoritaires dans la base et l'émetteur, alors que l'augmentation de $R_{\mathrm{p}}$ traduirait en partie la passivation des interfaces recombinantes.

Les incréments importants $\Delta \bar{J}_{\mathrm{ph}}$ exprimés en terme d'augmentation de longueur de diffusion effective, $L$ eff, des porteurs minoritaires correspondraient à des variations de $L$ eff de 50 à environ $100 \mu \mathrm{m}$ dans le cas du ruban E, tableau II. A l'instar du traitement PLH-430, le traitement IMPL-H se traduit par une forte augmentation de la longueur de diffusion, $L n$, dans les parties homogènes et une réduction de la vitesse de recombinaison sur les interfaces comme on peut le déduire d'enregistrements du photocourant local en mode LBIC [9].

L'étude de la forme des courbes de sensibilités spectrales, $S(\lambda)$, suggère par ailleurs que la "profondeur passivée " pour un traitement de 2 min à environ $175^{\circ} \mathrm{C}$ est de l'ordre de grandeur de l'épaisseur des échantillons, inférieure à $100 \mu \mathrm{m}$. Cette observation est corroborée par les résultats d'expérienses d'homogénéisation de la concentration en hydrogène dans l'épaisseur par traitements thermiques à $230^{\circ} \mathrm{C}$ sur des périodes de 0 à $60 \mathrm{~min}$, qui se tra- duisent par des augmentations relatives de $J_{\mathrm{ph}}$ toujours inférieures à $2 \%$. On notera que l'ordre de grandeur de la pénétration ainsi évaluée est en bon accord qualitatif avec celle déduite d'une'extrapolation aux basses températures des mesures de $\mathrm{A}$. Van Wierigen et N. Warmoltz [14].

3.2 Passivation des défauts ÉTendus. - Parmi les défauts étendus, les joints de grains et de mâcles sont avec les lignes de dislocations les défauts les plus fréquents dans le matériau RAD dont la texture cristallographique a été décrite par ailleurs $[5,15,16]$. Un travail récent a établi pour ce matériau une corrélation étroite entre la structure des joints déterminée par microscopie électronique en transmission (MET) et leur activité électrique qualitativement évaluée au MEB en mode EBIC [5]. Trois types de joints ont été identifiés : les mâcles en relation de coïncidence, $\Sigma=3^{n}$, les sous-joints du type $\Sigma=1, \Delta \theta$ et $\Sigma=3$, $\Delta \theta(\Delta \theta$, écart angulaire à la coïncidence parfaite, est en général inférieur au degré), et les joints quelconques. Selon cette étude, l'activité électrique de ces interfaces est en relation étroite avec leur densité de marches et (ou) de dislocations de matrice "absorbées ». Ainsi, les joints de mâcles symétriques $\Sigma 3$ exempts de dislocation de matrice, qui prédominent dans ce matériau, sont électriquement inactifs alors que les sous-joints $\Sigma=1, \Delta \theta$ et $\Sigma=3, \Delta \theta$ associés à des densités de dislocations de matrices et de marches élevées sont très actifs. Les micrographies MEB (Figs. 11 à 13) montrent que t'activité étectrique des interfaces recombinantes est considérablement réduite. par la passivation à l'hydrogène, au moins sur la profondeur "active " en EBIC, de l'ordre de $10 \mu \mathrm{m}$ pour la tension d'accélération des électrons primaires utilisée $(25 \mathrm{kV})$. Les images EBIC ont été réalisées avant et après IMPL-H pour établir la cartographie des défauts actifs, et les images de morphologie en électrons secondaires (ES) ont été faites après décoration de la surface au réactif de SIRTL afin de révéler les intersections des défauts avec la surface; l'échantillon décrit ici présentait une densité exceptionnelle de dislocations et d'interfaces $\Sigma 1, \Delta \theta$ et $\Sigma 3$ recombinantes.

Le rôle des dislocations sur la recombinaison aux joints de mâcles simples $\Sigma 3$ (notés 3 dans les micrographies) apparaît dans les figures 11 et 12 qui présentent respectivement des alignements de mâcles $\Sigma 3$ à interfaces symétriques avec et sans dislocations (les dislocations alignées dans les interfaces sont révélées par les puits d'attaque (Fig. 11a)).

On note après la passivation hydrogène une activité électrique très faible sur ces défauts y compris aux extrémités notées 3 e (Fig. 12a, b); ces extrémités ont en général une structure complexe caractérisée par une densité de marches élevée et la présence de microprécipités $[5,15]$.

Les sous-joints $\Sigma 1, \Delta \theta$ (et $\Sigma 3, \Delta \theta$ ), sont fréquemment observés dans les zones de cristallisation à isothermes planes à l'intérieur de grains de grande 


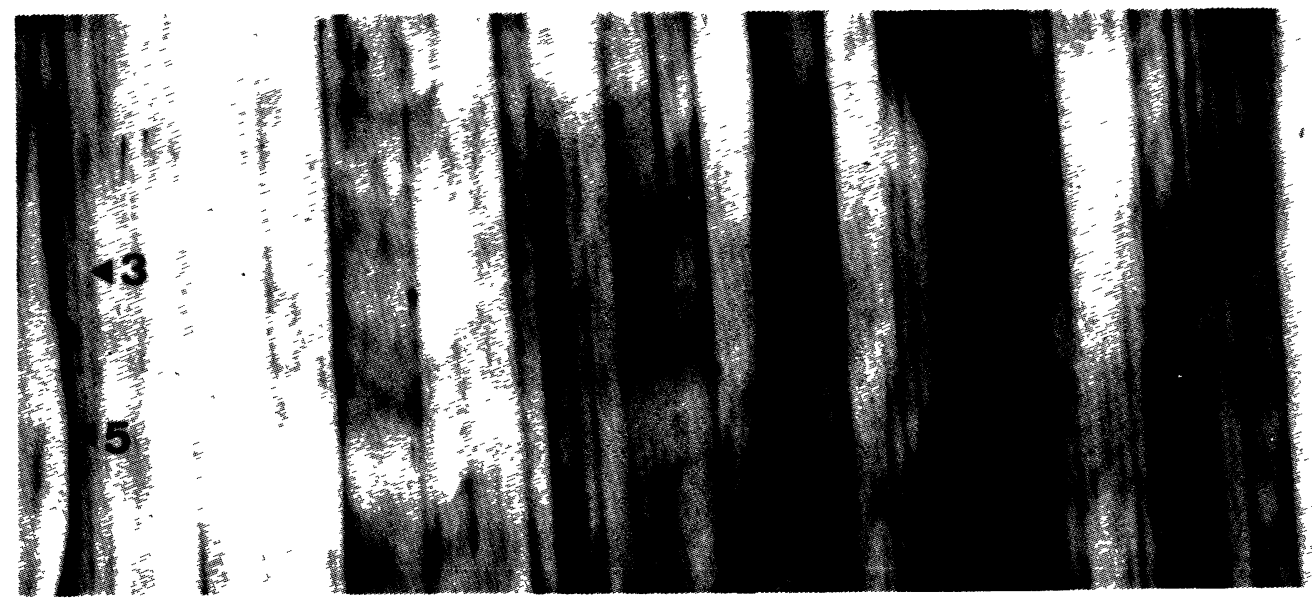

(a)

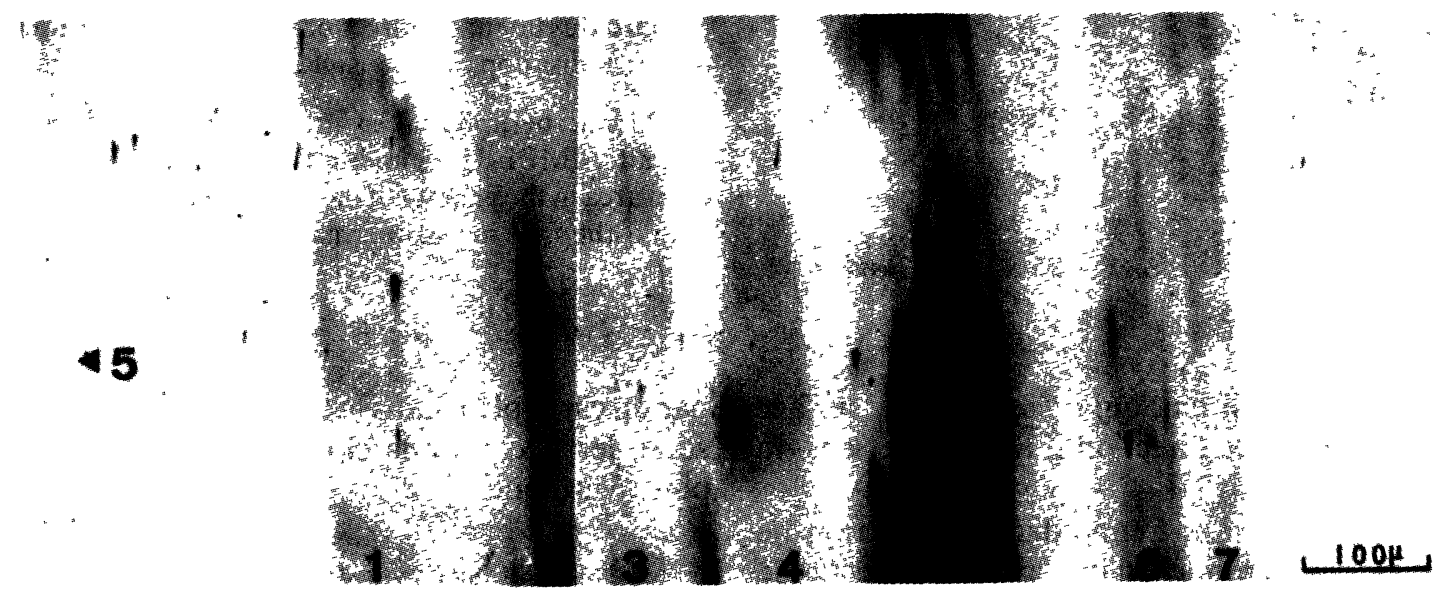

(b)

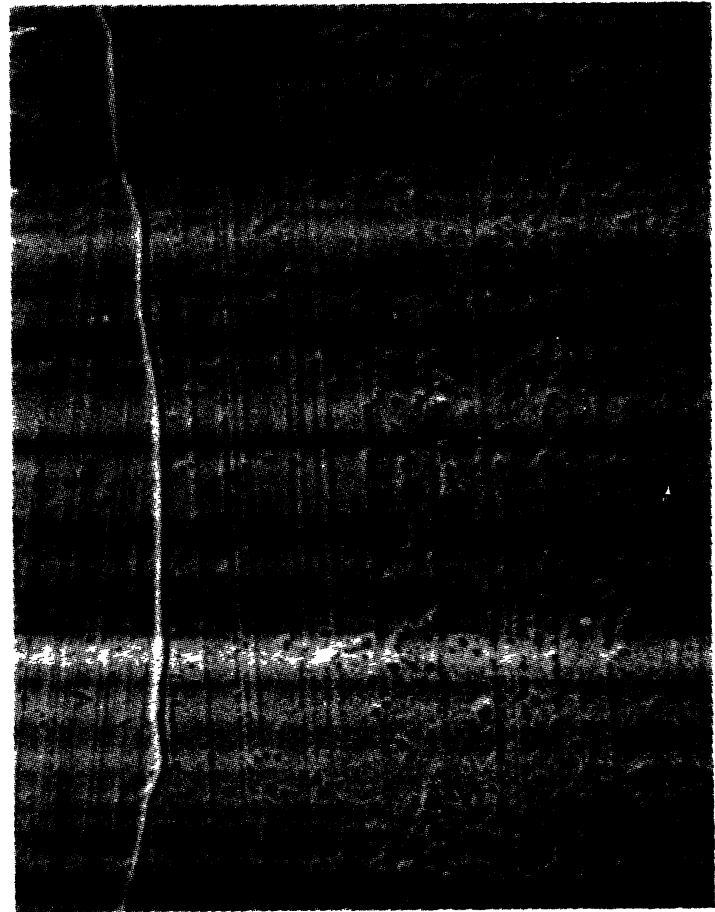

(c)
Fig. 11. - Micrographies MEB en mode EBIC avant (a) et après IMPL-H (b) et en mode ES (c) d'une zone riche en joints du type $\Sigma 1, \Delta \theta$ (2) et $\Sigma 3$ (3) recombinants; le joint (5) est un joint de faible désorientation. Les « bandes » caractérisées par une recombination élevée et délimitées par des interfaces $\Sigma 3$ sont numérotées 1 à 7 (b); la micrographie (c) est un grandissement de la zone du joint (5) qui met en évidence les alignements préférentiels des puits d'attaque dans certains joints $\Sigma 3$.

[SEM micrographs taken in the EBIC mode, prior to (a) and after IMPL-H (b) and in the electron emission mode (c) of an area showing recombining boundaries of the $\Sigma 1$, $\Delta \theta$ (2) and $\Sigma 3$ (3) types; boundary noted 5 is a low-angle boundary. "Strips " exhibiting a large electrical activity delineated by $\Sigma 3$ interfaces, are numbered 1 to 7 (b) ; micrograph (c) is an enlarged picture of the region of grain boundary 5 which shows etch pits preferentially aligned along some $\Sigma 3$ interfaces.] 


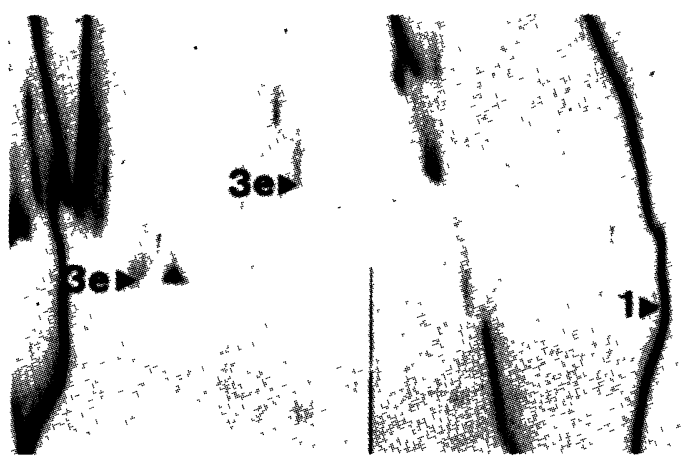

(a)

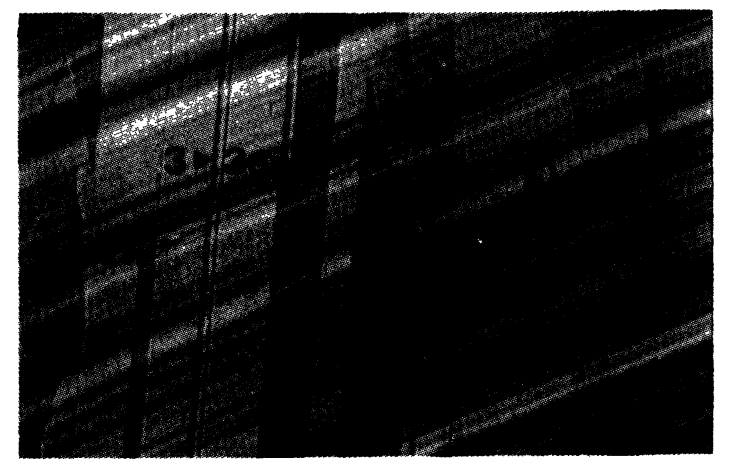

(b)

largeur (de l'ordre de $1 \mathrm{~cm}$ ) qui présentent une surface proche de (110) et une direction [112] dans le sens du tirage [15].

Ils se propagent en moyenne dans la direction de tirage et sont environnés d'une grande densité de défauts, dont des lignes de dislocations, approxinativement contenus dans une bande délimitée par des interfaces $\Sigma 3$ très actives; de telles bandes notées 1 à 7 accompagnant des sous-joints de type $\Sigma 1, \Delta \theta$ (notés 2) ou $\Sigma 3, \Delta \theta$ sont parfaitement visibles dans les micrographies EBIC de la figure 11. Compte tenu de leur activité électrique, ces sous-joints de structure cristallographique complexe déterminent en fait la largeur effective des zones homogènes des grains. La micrographie EBIC $11 \mathrm{~b}$ réalisée après IMPL-H met en évidence une forte réduction de l'activité électrique dans ces bandes où la recombinaison résiduelle est relativement homogène, et une passivation presque totale des interfaces des mâcles $\Sigma 3$.

Le joint de grains 5 de la figure 11 est aussi relativement fréquent dans les dépôts RAD; les mâcles $\Sigma 3$ de part et d'autre de ces joints font un angle voisin de $77^{\circ}$; ils pourraient être décrits comme une interface $\Sigma 3^{5}$ imparfaite [5]. L'activité électrique de tels joints, probablement liée à leur structure facettée, est elle aussi très réduite après IMPL-H, leur contraste EBIC ayant disparu.

On note encore sur ces micrographies la passivation quasi complète de plusieurs joints typiques des zones de bord de ruban où les isothermes(I) de cristallisation sont très courbes (Fig. 12) et une activité résiduelle forte dans la zone adjacente d'un joint (1) dénotée

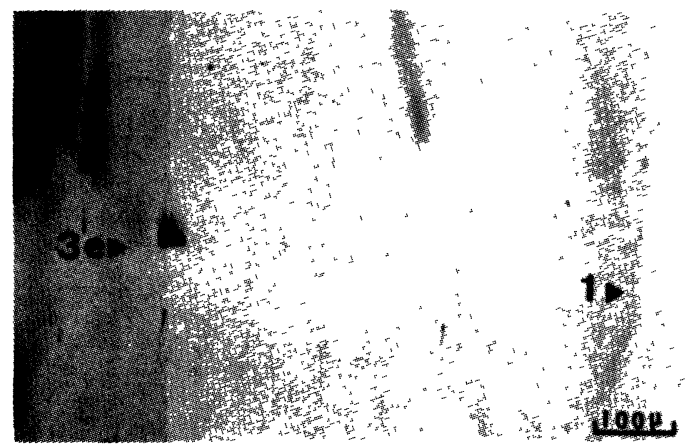

(c)

Fig. 12. - Micrographries MEB en mode EBIC avant (a) et après IMPL-H (b) et en modes ES (c) d'une zone proche du bord du ruban. La direction du tirage, $u$, les isothermes (I) de cristallisation sont visibles dans la micrographie (c).

[SEM micrographs taken in the EBIC mode, prior to (a) and after IMPL-H (b) and in the electron emission mode (c) of an area in the vicinity of the ribbon edge. The pull direction, $u$, and the freezing isotherms (I) are visible on micrograph (c).]

par un carré plein dans la figure 13; dans cette dernière zone, les décorations chimiques n'ont pas révélé de puits d'attaque.

3.3 Discussion. - La passivation chimique par l'hydrogène des défauts recombinants d'interfaces ou de volume est en accord qualitatif avec les observations faites en autoradiographie à haute résolution sur lames minces préparées sur des échantillons initialement chargés en tritium à $150^{\circ} \mathrm{C}$ par une méthode électrolytique [17]. Ces travaux avaient montré une ségrégation de tritium très importante le long des joints de grains et sur les dislocations extrinsèques dans les joints de mâcles $\Sigma 3$; une ségrégation en amas avait été aussi observée dans le volume exempt de défauts étendus. La présente étude a mis en évidence une passivation incomplète de zones sans défauts étendus, adjacentes à des joints de faible désorientation; cet effet suggère la présence de défauts recombinants autres que les liaisons pendantes (ou reconstruites) des coeurs de dislocations ou d'interfaces. Les impuretés ségrégées lors de la cristallisation pourraient être à l'origine de ces centres recombinants non passivés.

Du point de vue des performances globales des photopiles, les améliorations de leurs caractéristiques consécutives au traitement IMPL-H résultent de la réduction de l'activité des pièges de volume et dans une moindre mesure de la passivation des interfaces recombinantes compte tenu de leur éloignement moyen [4]. La nature des pièges de volume, étudiés jusqu'ici d'un point de vue phénoménologique seulement, 


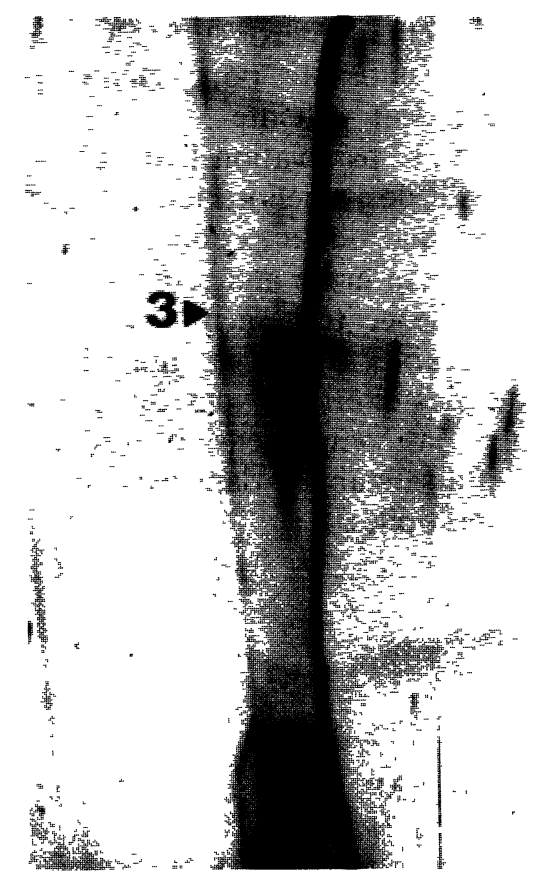

(a)

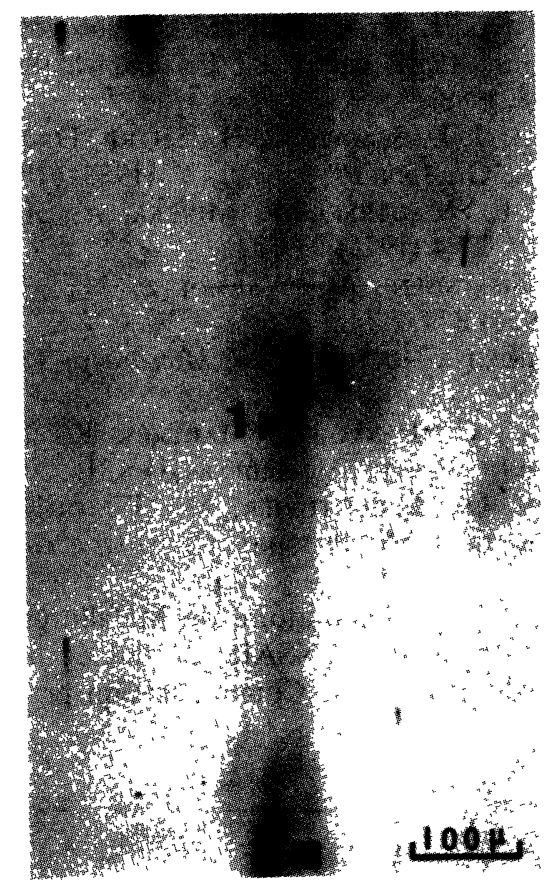

(b)

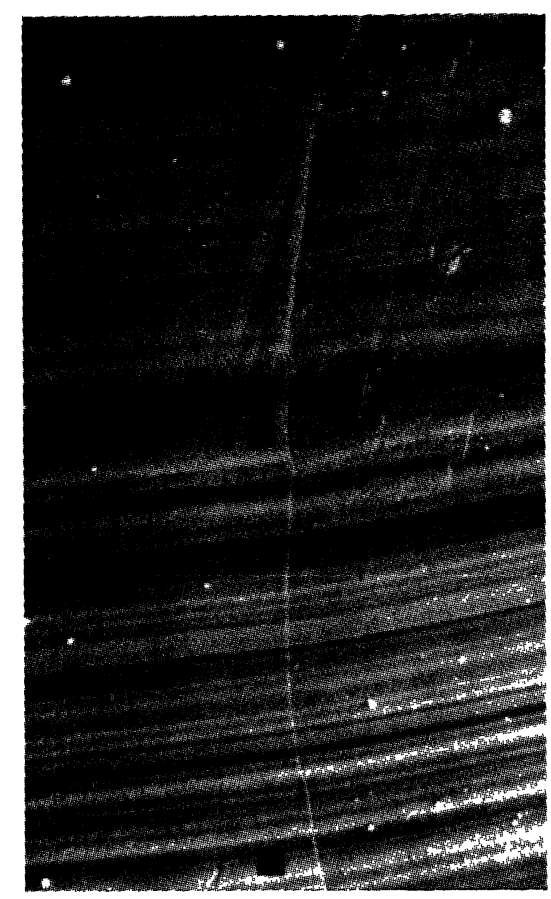

(c)

Fig. 13. - Micrographies MEB en mode EBIC avant (a) et après IMPL-H (b) et en mode ES (c). Le carré plein montre la zone adjacente au joint (1) non passivée après IMPL-H.

[SEM micrographs in the EBIC mode, prior to (a) and after IMPL-H (b) and in the electron emission mode (c). The full square denotes the region next to boundary (1) not passivated after IMPL-H.]

doit être élucidée afin d'en déterminer l'origine et l'aptitude à la passivation par l'hydrogène. Sur la base des résultats acquis, des rendements de conversion AM1 relativement élevés pourraient être atteints avec la maîtrise de l'hydrogénation au moyen d'une source de type Kaufman. Un rendement de 15,5\% $\left(V_{\text {co }}=559 \mathrm{mV}, J_{\mathrm{ph}}=37 \mathrm{~mA} / \mathrm{cm}^{2}, F F=0,75\right)$ a déjà été obtenu pour une photopile RAD de $3 \mathrm{~cm}^{2}$ et une valeur de $14,5 \%$ a été rapportée dans le cas de photopiles préparées sur ruban EFG et traitées IMPL-H [12]. Une augmentation du rendement des photopiles RAD devrait aussi résulter d'un meilleur

contrôle de la périphérie de jonction qui dégrade en général la tension de circuit ouvert et le facteur de forme. Enfin, la contamination de la surface de certains dépôts par des impuretés métalliques, par exemple le fer détecté en ESCA dans le cas du ruban F [18], pourrait être à l'origine des valeurs faibles de $V_{\text {co }}$ et $F F$ typiques de certains rubans. La stabilité des performances des photopiles ainsi passivées en fonction des paramètres temps, température et éclairement sera déterminante pour la viabilité du traitement IMPL-H. Cet aspect fait l'objet d'une étude systématique en cours. 


\section{Conclusion.}

La passivation par l'hydrogène des défauts recombinants dans les rubans de silicium polycristallin obtenus par la méthode RAD au moyen d'une source Kaufman se traduit par une augmentation importante du rendement de conversion des photopiles et un resserrement des distributions de leurs caractéristiques. Ces améliorations sont attribuables à une réduction de la vitesse de recombinaison aux interfaces et à une augmentation de la longueur de diffusion dans les parties homogènes. Ces études exploratoires ont montré qu'en dépit de traitements courts à basse température $\left(2 \mathrm{~min}\right.$ à environ $\left.175^{\circ} \mathrm{C}\right)$, la mobilité de l'hydrogène est suffisante pour qu'une passivation en profondeur soit atteinte.

L'efficacité de la passivation hydrogène aussi bien du point de vue de l'amélioration des performances des photopiles que de sa productivité, encore perfectible, notamment grâce aux sources d'ions du type Kaufman, fait de ce traitement une approche prometteuse applicable à tous les matériaux polycristallins. Les études ultérieures devront être consacrées notamment à la compréhension du processus de passivation et à sa stabilité dans les conditions d'utilisation des photopiles.

\section{Bibliographie}

[1] Wolf, M., Goldman, H. M. and Lawson, A. C., Spec. Conf., IEEE Cat no 78 CH 1319-3 ED (Washington D.C.) 1978, 271.

[2] Consulter par exemple : Shaped Crystal Growth. Ed. : Cullen, G. W., SureK, T., ANTonov, P. I. (North Holland Publishing Company, Amsterdam) 1980.

[3] Belouet, C., Texier-Hervo, C., Mautref, M., Belin, C., Paulin, J. et Schneider, J., J. Crystal Growth 61 (1983) 615.

[4] Belouet, C., Fabre, E., Makram-Ebeid, S., Ngo Tich Phuoc et Texier, C., Proc. 2nd E.C. Photovoltaic Solar Energy Conf., West Berlin (D. Reidel Publishing Company) 1979, p. 114.

[5] Sharko, R., Etude par microscopie électronique en transmission de joints de grains dans les couches de silicium polycristallin obtenues par la méthode $R A D$, Thèse de 3e cycle, juin 1983, Université Paris 6, France.

[6] Cambell, D. R., Brodsky, M. H., Hway, J. C. M., Robinson, A. E. et Albert, M., Bull. Am. Soc. 24 (1979) 435.

[7] Seager, C. H., Ginley, D. S., Appl. Phys. Lett. 34 (1979) 337.

[8] Seager, C. H., Sharp, D. J., Panitz, J. K. G. and HANOKA, J. I., J. Physique Colloq. 43 (1982) C1-103.
[9] Aucouturier, M., Rallon, O., Mautref, M., BeLOUET, C., Ibid. p. C1-117.

[10] Ginley, D. S., Haaland, D. M., Appl. Phys. Lett. 39 (1981) 271.

[11] Belouet, C., Hervo, C., Mautref, M., Fages, C., Hervo, J., Proc. 15th IEEE Photovoltaic Spec. Conf., IEEE cat $\mathrm{n}^{\circ} 82 \mathrm{CH}$ 1821-8 (San Diego, 1982), 80.

[12] Hanoka, J. I., Seager, C. H., Sharp, D. J., Panitz, J. K. G., Appl. Phys. Lett. 427 (1983) 618.

[13] Fabre, E., Mautref, M., Mircea, A., Appl. Phys. Lett. 274 (1975) 239.

[14] Van Wherhaen, A., Warmottz, N., Physica 22 (1956) 849.

[15] Fontaine, C., Rocher, A., J. Microscopy 1181 (1980) 105.

[16] Belouet, C., Hervo, J., Martres, R., Ngo Tich Phuoc, Pertus, M., same as [1], 131.

[17] Rallon, O., Aucouturier, M., Texier-Hérvo, C., Mautref, M., Belouet, C., Solar Cells 9 (1983) 149.

[18] Deville, J. P., Laboratoire de Métallurgie, Chimie des Matériaux, ENSAIS, 24, bd de la Victoire, 67000 Strasbourg, Communication privée. 